TITRE: COMPRENDRE LA MARGINALITÉ OU RENFORCER LA MARGINALISATION?

RÉFLEXIONS ÉTHIQUES AUTOUR D'UNE RECHERCHE

Auteur(s): ÉRIC GAGNON

Publication: RECRUTEMENT ET CONSENTEMENT À LA RECHERCHE : RÉALITÉS ET DÉFIS ÉTHIQUES

PAgES: $98-105$

ISBN: 978-2-7622-0357-8

Directeurs: AnA MARIN, BÉATRICE EySERMANN ET MiChEL T. GIROUX

URI: HTTP://HDL.HANDLE.NET/11143/14114

DOI: HTTPS://DOI.ORG/10.17118/11143/14114 


\title{
Comprendre la marginalité ou renforcer la marginalisation? Réflexions éthiques autour d'une recherche
}

\author{
Éric Gagnon, chercheur, Centre de recherche sur les soins et les services de première ligne de l'Université Laval, Centre intégré uni-
} versitaire de santé et de services sociaux de la Capitale-Nationale

Résumé : Sur la base d'une expérience de recherche portant sur la marginalisation et l'exclusion de certaines catégories de personnes des espaces publics, l'auteur soulève un ensemble d'interrogations éthiques. La recherche participe des débats politiques et sociaux touchant la marginalité et les marginaux, leur réalité et les problèmes qu'ils occasionnent. Ce faisant, elle risque, bien malgré elle, de contribuer à créer et à renforcer la marginalisation, et de renforcer l'image négative que les personnes ont d'elles-mêmes. L'auteur avance quelques propositions pour prévenir la violence que la recherche pourrait ainsi produire ou contribuer à alimenter.

Mots clés : Marginalisation, identité, débats, violence, sujet. 
Il y a quelques années, dans le cadre d'une recherche sur le partage des espaces publics dans un quartier de la basse-ville de Québec, j'ai mené des entrevues avec des personnes dites marginales. Or, très souvent ces personnes me parlaient des marginaux à la troisième personne; elles ne s'identifiaient pas à la marginalité. D'ailleurs, les entretiens n'étaient parfois possibles que dans la mesure où elles n'étaient pas directement associées aux marginaux. Ces personnes avaient pourtant été recrutées par des intervenants de groupes communautaires œuvrant auprès des sans-abri, des prostituées, des pauvres et des toxicomanes. Elles devaient nous donner le point de vue des personnes marginalisées sur la manière dont elles cohabitent et interagissent avec les autorités et les autres groupes présents dans le quartier - marchands, résidents, policiers - de même que sur leur place dans les espaces publics, les parcs, le parvis de l'église, les devantures des commerces (Gagnon et coll, 2016; Parazelli et coll., 2013).

Pourquoi cet écart entre le statut de marginal qu'on leur attribuait et l'identité qu'elles se reconnaissaient? Sans doute ne voulaient-elles pas être associées à un groupe stigmatisé et à une condition dont elles cherchaient à échapper. Personne n'aime être identifié à des conduites mal perçues et des groupes ostracisés. Peut-être l'entretien leur permettait-il également de prendre une distance avec leur situation et de l'analyser : en parler à la troisième personne aurait été une manière de prendre du recul. Ce qui est certain, c'est que parler de la marginalité n'est jamais neutre. C'est jeter un regard sur une condition définie par la négative, l'isolement, le rejet, l'étrangeté, par des comportements condamnés pour leur caractère inconvenant ou menaçant. Si nous pouvons prendre une certaine distance avec le phénomène, nous ne pouvons jamais nous en détacher entièrement. La marginalité est en effet largement le produit du regard que l'on porte sur elle : le regard que les individus portent sur certaines conduites, modes de vie ou apparences; le regard que l'individu, ainsi regardé et jugé, pose sur lui-même. La marginalité est un jugement porté par un groupe sur un autre groupe, c'est l'image que des individus, par défi ou en réaction à ce jugement, renvoient aux autres. C'est être regardé ou être regardant, ou encore regardant-regardé. On ne peut en parler sans être pris dans ce jeu des regards, sans devoir se situer en dehors ou au-dedans de la marginalité, sans prendre position ni juger.

Pour la personne sollicitée par le chercheur, accepter de participer à une étude sur les marginaux signifie savoir qui sont les marginaux et ce qui fait leur marginalité, accepter d'en discuter, de clarifier ses idées sur la question, de porter un jugement, et donc de se compromettre. Pour certains participants c'est encore plus engageant, cela signifie accepter ou rejeter l'étiquette de marginal ou d'itinérant; il ne s'agit plus seulement de savoir ce qu'est la marginalité, mais de savoir qui l'on est ou qui l'on veut être, à ses propres yeux et aux yeux des autres. Les participants à l'étude sont tous personnellement concernés, mais de manières différentes; ils sont tous regardants et regardés, mais les conséquences sur leur vie ne sont pas les mêmes.

S'il est une question éthique importante dans les études sur la marginalité, c'est celle du respect de l'intégrité morale des personnes. La recherche ne doit pas leur renvoyer une image négative d'elles-mêmes, elle ne doit pas contribuer à leur exclusion, leur dévalorisation, leur sentiment d'étrangeté, leur expulsion des espaces publics ou leur silence. Si les principes d'autonomie et d'intégrité de la personne s'appliquent en ce domaine, ce n'est pas uniquement par l'obtention du consentement ou par la préservation de l'anonymat, mais dans le maintien chez ces personnes d'une capacité de prendre la parole, d'exprimer un point de vue et de s'adresser aux autres sans honte, ou sans provoquer chez les autres un sentiment de honte. Le chercheur ne peut pas ne pas s'interroger sur les répercussions de son étude sur les marginaux, sur la manière dont on les regarde et la manière dont ils se perçoivent. Il est pris dans le jeu des regards. 
La marginalité, comme tous les objets de la sociologie et des sciences sociales en général, fait débat. Avant que le sociologue ne s'en empare, elle fait l'objet de controverses. Elle est largement discutée et débattue dans les médias, les ministères, au Parlement; de nombreux groupes et individus se sont déjà prononcés sur sa nature, ses causes, ses conséquences et les mesures à prendre pour la contrer. C'est une réalité déjà largement interprétée (Gagnon, 2009). Le sociologue s'invite dans un débat qui a commencé avant lui et qui va se poursuivre après lui. Il ajoute son interprétation à celles qui circulent déjà, une interprétation qu'il veut plus large et plus complète, mieux fondée et argumentée ${ }^{1}$, et qui relance la discussion. Il se jette à son tour dans la mêlée.

L'objet «marginalité» ne va pas de soi. Sa définition, ses frontières et sa signification sont débattues, sa nature même est polémique. L'objet du sociologue n'est d'ailleurs pas tant la marginalité en soi que le débat sur la marginalité. L'étude à laquelle j'ai fait allusion plus haut avait précisément pour objet un débat : le partage des espaces publics entre les personnes marginalisées et les autres populations fréquentant ou habitant le même quartier. Nous devions apporter un éclairage à une controverse qui existait déjà : les commerçants se plaignaient de la présence de certaines personnes dites «marginales» à proximité de leur commerce, des organismes communautaires prenaient la défense de ces personnes, les autorités municipales tentaient d'arbitrer le différend entre les commerçants et les groupes, et la police menait diverses interventions, tantôt répressives, tantôt apaisantes. Tout un chacun avait son explication quant à la nature et la source du problème, et le gouvernement provincial, qui avait financé notre étude, était à la recherche de solutions. Étudier la marginalité, ici comme ailleurs, c'est d'abord rendre compte d'un débat, c'est faire entendre des voix, rendre visibles des conduites, faire apparaître des jugements; en un mot : confronter les divers regards et poser à son tour un regard.

Dans une étude sur la marginalité, les questions épistémologiques, éthiques et politiques sont ainsi inextricablement liées. En définissant la marginalité, en cherchant à circonscrire le phénomène et à le caractériser, le chercheur et ses interlocuteurs (ou informateurs) sont conduits simultanément à se prononcer:

1. sur la perspective dans laquelle la marginalité doit être comprise, sa nature, ses causes, ses conséquences (questions épistémologiques);

2. sur l'identité du marginal, ce à quoi on le reconnaît, et donc sur le regard que l'on pose sur lui - son image, son égale dignité, le respect qu'on lui doit, l'importance accordée à son point de vue (questions éthiques);

3. sur les politiques à adopter - répressives ou pas -, sur les règles à faire respecter et la place accordée aux conduites marginales dans la société (questions politiques).

1. C'est ce qui différencie une interprétation sociologique d'une idéologie : comme tout discours scientifique, l'interprétation du sociologue se veut argumentée et son auteur accepte de la soumettre à la critique en exposant ses arguments et en se soumettant à l'évaluation par les pairs (colloques et publications). Elle prétend également rendre compte d'un plus grand nombre de dimensions du phénomène étudié que les autres discours. 
Les questions épistémologiques, éthiques et politiques ne se séparent pas; elles sont plutôt les trois faces d'une même préoccupation ou interrogation : Quels sont les rapports que les divers groupes sociaux entretiennent avec la marginalité et le marginal, quels sont les rapports qu'ils devraient entretenir avec eux?

La question du respect de l'intégrité morale des participants à la recherche s'inscrit dans une question éthique aux plus larges dimensions touchant la participation du chercheur et des participants à un débat moral et politique. La question n'est pas simplement déontologique (préserver l'anonymat des personnes dans le recueil des données et l'exposé des résultats), elle consiste à entrer de plain-pied dans un débat public, y faire entrer d'autres personnes - les marginaux, les commerçants, les résidents - en faisant entendre leur voix, en suscitant leur réflexion et en les obligeant à se regarder, à mettre en lumière les différentes dimensions du problème. La question éthique pour le chercheur est de décider à qui il va donner la parole, comment il va la donner et ce qu'il va faire de ce qu'il a entendu.

\section{III}

Cette question, aux dimensions tant épistémologiques, éthiques que politiques, se pose au chercheur à toutes les étapes de son étude.

1. Elle se pose au tout début de son enquête au moment de formuler sa question de recherche. Déjà, il lui faut retenir certains critères par lesquels il reconnaît la marginalité afin de pouvoir identifier des conduites sur lesquelles il interrogera diverses personnes. Même s'il se garde alors de porter explicitement un jugement sur ces individus ou ces conduites, il les désigne comme faisant problème pour d'autres. Il sélectionne parmi différents problèmes débattus dans la société ceux qui doivent retenir l'attention, et cela pourra inclure autant les conduites marginales que les conduites de réprobation ou d'exclusion à l'endroit des marginaux. La manière dont le problème est formulé et la perspective retenue (et conséquemment d'autres perspectives rejetées) reposent sur des choix théoriques, mais aussi éthiques et politiques sur ce qui mérite attention, et qui ne seront pas sans conséquence sur le regard que les participants seront amenés à poser sur les autres et sur eux-mêmes.

2. Cette question éthique demeure présente au moment d'approcher et de recruter des personnes pour un entretien ou répondre à un questionnaire. Comment faut-il les aborder? À quel titre le chercheur va-t-il leur demander de participer à l'étude? En tant que personne marginalisée, ou à titre d'usager d'un centre d'aide pour toxicomanes, sans préjuger de leur marginalité? En tant que résident ou commerçant du quartier ou comme personne demandant l'intervention de la police ou l'adoption de nouvelles réglementations municipales visant à réduire la présence des marginaux? À quel titre, en vertu de quelles connaissances ou de quelle compétence, au nom de quels intérêts ou de quelle autorité vont-elles parler? Quelle position dans le débat leur demandera-t-il d'occuper? Cette position ne sera pas sans conséquence sur la légitimité de leurs discours et de leur participation au débat.

3. Dans la manière dont les entretiens seront conduits, la question va continuer de se poser. Les thèmes retenus, la formulation des questions, jusque dans l'attitude et les réactions de l'intervieweur face aux propos tenus par la personne interrogée, auront une incidence - minime parfois, forte à d'autres moments - sur la manière dont cette personne va se percevoir et se sentir jugée, sur sa compréhension du problème, sur la légitimité de certaines conduites, la sienne, celle des autres (autant la conduite marginale que la conduite répressive ou d'exclusion). Comprenons bien ici que le fait qu'un individu soit amené à réfléchir sur sa conduite, à la voir autrement, à se remettre en question n'est pas en soi un problème. Nous avons affaire à des adultes qui ont librement consenti à discuter d'un problème et à répondre à des questions. C'est souvent même l'un 
des objectifs de l'étude que de susciter pareille remise en question, tant chez les marginaux que chez les commerçants ou les policiers. Après tout, il s'agit de faire avancer le débat. Le chercheur doit cependant savoir que ce qu'il fait n'est pas neutre ni sans effet, et que, si ces effets ne sont pas entièrement contrôlables, il lui faut minimiser les plus indésirables (honte, gêne, mutisme).

4. Enfin, cette question se pose au moment de communiquer les résultats de la recherche lorsqu'il s'agit de choisir ses mots pour une conférence ou un article. Comment décrire la situation, rendre compte du débat, présenter les positions et caractériser les comportements de chacun, sachant qu'il n'y a pas de manière neutre de le faire? Quelle image le chercheur laisse-t-il des individus? Comment fera-t-il droit à leur parole et à leur point de vue (sans nécessairement les endosser)? Il faut de toute évidence peser ses mots. Là encore, ça ne veut pas dire éviter toute réaction ou tout débat, au contraire, mais éviter de blesser ou de vexer inutilement. Il faut aussi être prêt à défendre sa lecture des évènements et des problèmes, montrer ce qu'elle ajoute, ce qu'elle permet de faire entendre ou de faire voir, sans pour autant faire taire d'autres voix ou masquer d'autres réalités. Elle doit élargir la compréhension du débat, accroître la vision, la portée des regards.

Le choix des mots, l'image ou l'interprétation produite nous ramènent au recrutement des sujets de recherche. Lorsqu'une personne marginalisée a accepté de répondre à nos questions, elle nous a fait confiance, elle a pensé que cela ne pouvait pas lui nuire, et peut-être que cela pourrait même améliorer sa situation. II en est de même pour le commerçant ou le résident qui se plaignent de la présence des marginaux. En acceptant de répondre à nos questions, il nous a fait confiance lui aussi : son point de vue et ses arguments allaient être entendus, pris au sérieux, sérieusement discutés, et non simplement écartés ou condamnés. Le chercheur s'est engagé à ne pas traiter ce dernier simplement comme une personne intolérante et répressive, aux comportements moralement condamnables. En le sollicitant, le chercheur a pris un engagement - explicite ou tacite - à respecter qui il est, ce qu'il pense et ce qu'il fait. Le chercheur ne peut l'oublier.

\section{IV}

Je terminerai par trois remarques. La première est que la recherche en sciences sociales peut être violente. Par les représentations des individus et des situations qu'elle produit ou reproduit, elle peut contribuer à entretenir un sentiment d'infériorisation, d'insignifiance ou d'impuissance, raviver aussi des blessures. Par les interprétations qu'elle avance, elle peut contribuer à réduire ou à durcir les différences, à renforcer ou à atténuer l'exclusion sociale, à donner la parole ou à imposer le silence. Comme tout regard, elle peut renvoyer aux autres une image négative d'eux-mêmes, leur enlever toute volonté ou toute capacité de prendre la parole, de se raconter ou d'agir, et ainsi réduire leur autonomie². Les savoirs peuvent être la source d'une réelle violence. Sans doute faut-il en prendre le risque. Se taire, ce serait faire perdurer une situation ou des préjugés. L'éthique ne consiste pas ici à tout faire pour éviter d'entrer dans le débat et de remuer les esprits, mais à y entrer prudemment.

La seconde remarque porte sur la perspective d'analyse. Plutôt que de se centrer sur les individus, il faut se centrer sur les rapports que ces individus entretiennent. Il faut étudier les processus de marginalisation plutôt que les marginaux; des processus qui se traduisent dans des jugements, des conduites d'évitement ou d'expulsion, de défi ou de mise à distance, mais aussi des représentations, des règlements et des aménagements urbains. Ce changement de perspective permet d'abord de ne pas fixer l'attention sur les individus, leur mode de vie ou leur conduite, et de prévenir les jugements trop rapides à leur endroit. II permet ensuite de faire avancer la réflexion, d’élargir la vision en se plaçant au niveau des rapports entre les individus plutôt que les individus

2. Je reprends la définition de l'autonomie proposée par Ricoeur (2001) qui se caractérise par la triple capacité de dire, de faire et de se raconter. 
considérés séparément. Ce déplacement du regard permet de penser l'altérité, d'interroger les différences et les distances que les différents groupes établissent entre eux, de questionner les écarts et les antagonismes que produisent ces différences, de comprendre l'inquiétude, les peurs et la méfiance que ces distances entretiennent. Ce déplacement permet également de prendre une distance avec nos manières de voir et de penser, d'avoir accès à d'autres points de vue, non pour les adopter et s'y installer, mais pour en saisir le sens et les raisons. Il permet de réduire l'altérité sans l'abolir, de réduire les différences en les rendant compréhensibles, sans pour autant les effacer.

La troisième remarque touche au débat social. En changeant la perspective, la sociologie contribue non seulement à élargir la compréhension du phénomène, mais également à élargir la participation au débat. Elle permet de donner la parole et d'écouter ce qui se dit, de faire entendre une pluralité de voix, au premier chef celles qu'on entend peu. Elle permet à une diversité de personnes et de groupes de participer à l'interprétation du problème, à sa compréhension et à sa transformation. Sans faire disparaître les divisions et les conflits, elle donne la possibilité à tous de faire entendre ses raisons, mais aussi d'opérer un déplacement, de modifier le regard qu'ils portent sur les autres. Le terme sujets pour désigner les participants à une étude prend alors tout son sens. Son emploi est rarement aussi significatif et conséquent que dans la recherche sur la marginalité et les marginaux. 


\section{Bibliographie}

Gagnon, É. (2009). Politique du cercle. Interprétation, éthique et sociologie, Cahiers de recherche sociologique, 48, 15-26.

Gagnon, É, M. Parazelli et Hardy, M.-H. (2016). Visibles et invisibles. Marginalités et partage des espaces publics à Québec. Dans D. Lamoureux et F. Dupuis-Déri (dir.) Au nom de la sécurité ! Criminalisation de la contestation et pathologisation des marges (p. 113-129), Montréal : M éditeur.

Parazelli M. et coll. (2013). Les enjeux du partage de l'espace public avec les personnes itinérantes et sa gestion à Montréal et à Québec. Perspectives comparatives et pistes d'actions. Fonds de recherche du Québec -Société et culture, Rapport de recherche Programme actions concertées, 19 août 2016, [version électronique]. http://www.frqsc.gouv.qc.ca/documents/11326/448958/PC_PazarelliM_rapport+2013_espaces+publics+vs+itinérance/

Ricœur, P. (2001). Le juste 2, Paris : Éditions Esprit. 